\title{
Non-Fermi liquid signatures in the Hubbard Model due to van Hove singularities
}

\author{
Sebastian Schmitt $^{1, *}$ \\ ${ }^{1}$ Lehrstuhl für Theoretische Physik II, Technische Universität Dortmund, \\ Otto-Hahn Str. 4, D-44221 Dortmund, Germany
}

(Dated: October 31, 2018)

\begin{abstract}
When a van-Hove singularity is located in the vicinity of the Fermi level, the electronic scattering rate acquires a non-analytic contribution. This invalidates basic assumptions of Fermi liquid theory and within treatments based on perturbation theory leads to a non-Fermi liquid self-energy and transport properties. Such anomalies are shown to also occur in the strongly correlated metallic state within dynamical mean-field theory. We consider the Hubbard model on a two-dimensional square lattice with nearest and next-nearest neighbor hopping within the single-site dynamical mean-field theory. At temperatures on the order of the low-energy scale $T_{0}$ an unusual maximum emerges in the imaginary part of the self-energy which is renormalized towards the Fermi level for finite doping. At zero temperature this double-well structure is suppressed, but an anomalous energy dependence of the self-energy remains. For the frustrated Hubbard model on the square lattice with next-nearest neighbor hopping, the presence of the van Hove singularity changes the asymptotic low temperature behavior of the resistivity from a Fermi liquid to non-Fermi liquid dependency as function of doping. The results of this work are discussed regarding their relevance for high-temperature cuprate superconductors.
\end{abstract}

\section{INTRODUCTION}

The question whether interacting fermions form a Landau Fermi liquid state at low temperatures or rather transform into something commonly referred to as singular or non-Fermi liquid is a very fundamental one. A theoretical description requires at least a qualitative understanding of the low temperature phase in order to address the relevant degrees of freedom in that phase. But for strongly interacting electronic systems this information is usually lacking, and the low-energy degrees of freedom might be very different from free electrons. However, the Fermi liquid picture is often appropriate at low temperatures and energies. Reasons for this can be found in the stability of the Fermi liquid fixed point of the renormalization group analysis ${ }^{1}$ or phase space arguments. ${ }^{2}$ In both approaches, the analyticity of the scattering vertices is crucial for the arguments.

In situations, where the Fermi liquid description fails, the origin for this is linked to low-dimensionality of the system, long-range interactions, nested Fermi surfaces or the proximity to a quantum critical point, just to name a few (see, e.g. Refs. 3-6). The quasiparticle concept might still be valid, but the scattering rate is strongly enhanced and shows an unusual, i.e. non-quadratic, temperature and frequency dependence. Alternatively the quasiparticle concept may also break down completely.

In this work we will focus on the single-band Hubbard model which is a minimal model for strongly correlated electron systems, and obtain a non-perturbative approximative solution by means of the dynamical mean-field theory (DMFT).$^{7,8}$ Within DMFT, where only local correlations are included, the generic low temperature state of the metallic Hubbard model is a Fermi liquid. ${ }^{8-13}$ This is expected, since the low temperature phase of the spin$\frac{1}{2}$ single impurity Anderson model - the model onto which the Hubbard model is mapped within DMFT - is a local Fermi liquid under the assumption of a "well behaved" medium. ${ }^{14}$ The Fermi liquid reveals itself via a low-energy many-body bandstructure forming around the Fermi level at low temperatures. ${ }^{15,16}$ Due to the neglect of momentum dependent correlations in DMFT the heavy quasiparticles possess the non-interacting Fermi surface. ${ }^{9}$ A characteristic low-energy scale $T_{0}$ is associated with this lattice version of the Kondo effect and marks the temperature scale for local-moment screening and the emergence of coherent quasiparticles.

On the other hand it is known from perturbation theory and renormalization group treatments, that a van Hove singularity of the non-interacting density of states (DOS) in the vicinity of the Fermi level lead to a marginal ${ }^{17}$ or non-Fermi liquid form of the selfenergy. ${ }^{18-31}$ This should be contrasted to nested Fermi liquids, ${ }^{3}$ where due to the nesting property of the Fermi surface the phase space volume for low-energy scattering is also strongly enhanced and unusual low-energy properties emerge as well..$^{3,32}$

Van Hove singularities are a consequence of maxima or saddle points in the dispersion relation $t_{k}$. For periodic energy bands in a crystal a certain number of these van Hove singularities must occur on topological grounds (see, e.g. Ref. 33). For example, all of the cubic lattices have noticeable van Hove singularities in space dimensions $d \lesssim 3$, typically logarithmic divergences or squareroot cusps.

Anomalous low-energy behavior can already be anticipated by recognizing, that the usual arguments of microscopic Fermi liquid theory break down if a maximum or saddle point of the dispersion relation $t_{\underline{k}}$ is found at the Fermi level. Then, $t_{\underline{k}}$ cannot be approximated by a linear dispersion, i.e. $t_{\underline{k}} \not \approx \frac{k_{F}}{m^{*}}|\Delta \underline{k}|$, with $\Delta \underline{k}=\underline{k}-\underline{k}_{F}$ and $\underline{k}_{F}$ the Fermi wave vector. Around these points a quadratic (maximum) or hyperbolic (saddle-point) functional dependence $t_{\underline{k}} \sim \Delta k_{\perp}^{2} \pm \Delta k_{\|}^{2}$ results, where $k_{\perp}$ and $k_{\|}$are 
two linear independent directions in momentum space. The arguments leading to a quadratic Fermi liquid energy and temperature dependence of the scattering rate, i.e. $\operatorname{Im} \Sigma^{U}\left(\omega-i 0^{+}\right) \sim \omega^{2}+\pi^{2} T^{2}$, are thus not applicable. Instead, the phase space volume for scattering is strongly enhanced and anomalous $T$ - and $\omega$-dependencies may result. If the singularity occurs in the vicinity of the Fermi level, strong modifications of the low-energy and temperature properties are still expected due to a pinning effect. ${ }^{26,34-38}$

In the present work we show that the non-Fermi liquid signatures which were encountered in previous studies $^{18-31}$ of the self-energy and transport properties can also occur in the paramagnetic phase of a strongly correlated metal within DMFT. We use the two-dimensional square lattice with nearest and nextnearest neighbor hopping, $t$ and $t^{\prime}$ respectively. For $-0.5<t^{\prime} / t<0.5$ the dispersion relation $t_{\underline{k}}$ has saddles at the $X$ points in the Brillouin zone $\left(\underline{k}_{X}=\right.$ $\{(0, \pm \pi),( \pm \pi, 0)\})$. This leads to a logarithmic divergence in the non-interacting DOS, which is located at the Fermi level $\omega=0$ for $t^{\prime}=0$ and moved to finite energies for $t^{\prime} \neq 0$.

The resulting anomalies consist of an unusual maximum in the imaginary part of the self-energy $\operatorname{Im} \Sigma^{U}(\omega-$ $i 0^{+}$) in the vicinity of the Fermi level at low but finite temperatures. The concept of quasiparticles remains valid but they are strongly scattered at the flat parts of the dispersion relation and the Fermi liquid formation is disturbed. The anomalous double well structure persists over a range of doping and next-nearest neighbor hopping $t^{\prime}$. At zero temperature and for half-filled square lattice the double-well structure is removed and the self-energy vanishes at the Fermi level. Even though the self-energy exhibits a non-Fermi liquid energy dependence, the quasiparticle weight is still finite. The pinning of the van Hove singularity to the Fermi level, which has previously been found, ${ }^{18,26,34-38}$ is explicitly observed in the correlated metal.

Qualitatively similar non-Fermi liquid signatures are also observed in more advanced theories which include nonlocal correlations. ${ }^{39-45}$ These features are usually attributed to the presence of strong nonlocal antiferromagnetic correlations. However, in the present approach nonlocal correlations are not included and we argue that an interpretation exclusively in terms of nonlocal correlations is too simplified.

The paper is organized as follows. In Section II we shortly present the Hubbard Hamiltonian and the basic equations of DMFT. We elucidate the role of the non-analyticities in the non-interacting DOS by means of a simplified model DOS for which analytical insights are obtained in Sect. III. Self-consistent DMFT calculations for singular and non-singular model DOS reveal the non-analyticity to be the cause for the observed nonFermi liquid signatures. In Sect. IV we present finite and zero temperature calculations for the spectral function and self-energy for a square lattice without next-nearest neighbor hopping $t^{\prime}=0$. The anomalies are shown to occur at temperatures on the order of the low energy scale $T_{0}$ and decrease with temperature. At $T=0 \mathrm{a}$ non-quadratic energy dependence in the imaginary part of the self-energy remains. The case of the square lattice with next-nearest neighbor hopping $t^{\prime}=-0.2 t$ is discussed in Sect. V. In addition to the spectral function and the self-energy, we also study the temperature dependence of the zero frequency quasiparticle scattering rate and the resistivity. We discuss in Sect. VI the relevance of the present findings to the cuprate superconductors before we conclude the paper.

\section{MODEL AND METHOD}

We study the single band Hubbard model

$$
\hat{H}=\sum_{i j, \sigma} t_{i j} \hat{c}_{i \sigma}^{\dagger} \hat{c}_{j \sigma}+\sum_{i \sigma} \epsilon \hat{c}_{i \sigma}^{\dagger} \hat{c}_{i \sigma}+U \sum_{i} \hat{n}_{i \uparrow} \hat{n}_{i \downarrow}
$$

where the operator $\hat{c}_{i \sigma}\left(\hat{c}_{i \sigma}^{\dagger}\right)$ annihilates (creates) an electron in a localized Wannier orbital at lattice site $i$ with spin $\sigma, \hat{n}_{i \sigma}=\hat{c}_{i \sigma}^{\dagger} \hat{c}_{i \sigma}$ represents the number operator for electrons, $\epsilon$ is the local ionic level position at each site, and $U$ is the local matrix element of the Coulomb repulsion.

In the following an external magnetic field is not included and all spin up and down quantities are equal. The spin label of Green's functions and self-energies will be suppressed. We focus on the paramagnetic phase of the model and exclude possible phase transitions to ordered phases such as superconductivity or magnetism.

All information on the lattice is encoded in the oneparticle hopping amplitude $t_{i j}$. Its Fourier-transform gives the dispersion relation

$$
t_{\underline{k}}=-2 t\left(\cos k_{x}+\cos k_{y}\right)-4 t^{\prime} \cos k_{x} \cos k_{y}
$$

where we set the lattice constant $a=1$ and already specified to the two-dimensional $(2 d)$ square lattice with nearest and next-nearest neighbor hopping, $t$ and $t^{\prime}$, respectively.

The central quantity for the discussion of Fermi liquid properties is the correlation self-energy $\Sigma^{U}(z)$. The assumption that it does not depend on momentum $\underline{k}$ constitutes the major approximation of DMFT. The lattice Green's function of the Hubbard model is then given by

$$
G\left(t_{\underline{k}}, z\right)=\frac{1}{z-t_{\underline{k}}-\Sigma^{U}(z)},
$$

which only depends on $\underline{k}$ via the dispersion relation $t_{\underline{k}}$. The latter also determines the non-interacting DOS $\rho_{0}(\omega)=\frac{1}{N} \sum_{\underline{k}} \delta\left(\omega-t_{\underline{k}}\right)$.

Within DMFT the lattice model is mapped onto an effective impurity model, where the medium $\Gamma(z)$ of the impurity model cannot be chosen arbitrarily, but has to 
be determined from the self-consistency conditions

$$
\begin{aligned}
\Gamma(z) & =\frac{T(z)}{1+\tilde{G}(z) T(z)} \\
T(z) & =\frac{1}{N} \sum_{\underline{k}} \frac{t_{\underline{k}}^{2}}{\tilde{G}(z)^{-1}-t_{\underline{k}}}=\int d x \frac{x^{2} \rho_{0}(x)}{\tilde{G}(z)^{-1}-x} \\
\tilde{G}(z) & =\frac{1}{z-\epsilon-\Sigma^{U}(z)} .
\end{aligned}
$$

The functional form of the local scattering matrix $T(z)$ is completely determined by the non-interacting DOS. The correlation self-energy enters only as an unspecified parameter [via $\tilde{G}(z)$ ] and is obtained from the solution of the effective impurity model for a given medium $\Gamma(z)$. The self-consistency cycle is closed by identifying the local Green's function of the lattice with the one of the impurity model,

$$
\begin{aligned}
G^{(\mathrm{loc})}(z) & =\frac{1}{z-\epsilon-\Sigma^{U}(z)-\Gamma(z)} \\
& \stackrel{!}{=} \frac{1}{N} \sum_{\underline{k}} G\left(t_{\underline{k}}, z\right)=\tilde{G}(z)+\tilde{G}(z) T(z) \tilde{G}(z)
\end{aligned}
$$

For the finite temperature calculations we use the enhanced non-crossing approximation ${ }^{15,16,46-48}$ (ENCA) as the impurity solver. There are no adjustable parameters and the method works directly on the real frequency axis. This is crucial for the observation of the small lowenergy anomalies in the self-energy. However, it cannot be employed at too low temperatures $T \ll T_{0}$ due to the NCA pathology. ${ }^{49}$ Within the ENCA the pathologic behavior is considerably improved compared to NCA. ${ }^{50}$ It can be reliably used down to temperatures $T \approx T_{0} / 10$ for moderate to large values of $U$ and not too far away from half-filling.

At zero temperature we use the numerical renormalization group ${ }^{51-53}$ (NRG) instead. For the NRG spectral functions we use the complete Fock-space ${ }^{54,55}$ algorithm of Ref. 56 with a discretization parameter $\Lambda=1.7$ and keep approximately 1700 states in each NRG-iteration step. We average over eight different discretizations ${ }^{57}$ of the conduction band to minimize the errors.

We also performed finite temperature DMFT(NRG) calculations which were in accord with the DMFT(ENCA) results and confirmed the existence of the anomalous structures in the self-energy. However, the anomalous features occur at energies and temperatures where the results depend on the actual values of NRG broadening parameters. ${ }^{56,58}$ Therefore, we prefer the ENCA for the finite temperature calculations.

\section{MODEL DENSITY OF STATES}

Before presenting DMFT results for the Hubbard model on cubic lattices we elucidate the basic mechanism by means of a simplified model DOS of the form

$$
\rho_{\alpha}^{\text {cusp }}(\omega)=\frac{1+\alpha}{2 \alpha W}\left[1-\left(\frac{|\omega|}{W}\right)^{\alpha}\right], \quad \alpha \geq 0
$$

with $-W \leq \omega \leq W$. For $\alpha<2$ this DOS has a nonanalytic cusp at $\omega=0$ which turns into a logarithmic divergence for $\alpha=0, \rho_{\alpha=0}^{\text {cusp }}(\omega)=\frac{1}{2 W} \ln \left(\frac{W}{|\omega|}\right)$, while it is smooth for $\alpha \geq 2$ (see inset of Fig. 1). The case $\alpha=0$ mimics the logarithmic divergence of the $2 d$ simple cubic lattice.

The explicit form (8) allows for the analytic calculation of the effective medium $\Gamma(z)$ as shown in the Appendix. We state the result for three values of $\alpha$

$$
\begin{aligned}
\Gamma_{\alpha=0}(z) & =\frac{1}{\tilde{G}(z)}+\frac{2 W}{\operatorname{Li}_{2}[-\tilde{G}(z) W]-\operatorname{Li}_{2}[\tilde{G}(z) W]} \\
\Gamma_{\alpha=1}(z) & =\frac{1}{\tilde{G}(z)} \\
& -\frac{W}{\frac{1}{G(z) W} \ln \left[1-(\tilde{G}(z) W)^{2}\right]+2 \operatorname{atanh}[\tilde{G}(z) W]} \\
\Gamma_{\alpha=2}(z) & =\frac{1}{\tilde{G}(z)} \\
& -\frac{1}{\frac{1}{\tilde{G}(z) W}+\left(1-\frac{1}{(\tilde{G}(z) W)^{2}}\right) \operatorname{atanh}[\tilde{G}(z) W]},
\end{aligned}
$$

where $\operatorname{Li}_{2}(z)$ is the Dilogarithm. The crucial difference in the analytic structure of the three results is the appearance of non-analytic logarithms for $\alpha=0$ and $\alpha=1$ which are absent for $\alpha=2$.

Suppose the system is about to form a Fermi liquid state as it is expected within DMFT for the Hubbard model at low $T$. Then, the correlation self-energy has the form,

$$
\omega-\epsilon-\Sigma^{U}\left(\omega-i 0^{+}\right)=\frac{1}{Z}\left(\omega-\tilde{\epsilon}-i \gamma\left[(\pi T)^{2}+\omega^{2}\right]\right),
$$

where $T$ is the temperature, $\gamma$ a measure for the residual quasiparticle scattering, and $Z$ the quasiparticle weight. The renormalized single-particle energy $\tilde{\epsilon}=$ $\epsilon+\operatorname{Re} \Sigma^{U}\left(-i 0^{+}\right)$determines the Fermi wave vector via $\tilde{\epsilon}=t_{\underline{k}_{F}}$ and vanishes in symmetric situations.

Using the form (12) in the expressions Eqs. (9) to (11), the imaginary part of $\Gamma$ develops a cusp-like minimum at the Fermi level for small $\gamma$ and $\alpha<2{ }^{59}$ Figure 1 illustrates this for the three different values of the exponent $\alpha=0,1$, and 2 . In the limit $\gamma \rightarrow 0$ the effective media for $\alpha=1$ and $\alpha=2$ approach the values $\operatorname{Im} \Gamma_{\alpha=1}=1 / \pi$ and $\operatorname{Im} \Gamma_{\alpha=2}=4 /(3 \pi)$, respectively, while for $\alpha=0$ the effective medium eventually reaches zero (see Eqs. (A.8) to (A.10)). Thus, for $\alpha=0$, we end up with a soft-gap effective medium for which Kondo screening and the local 


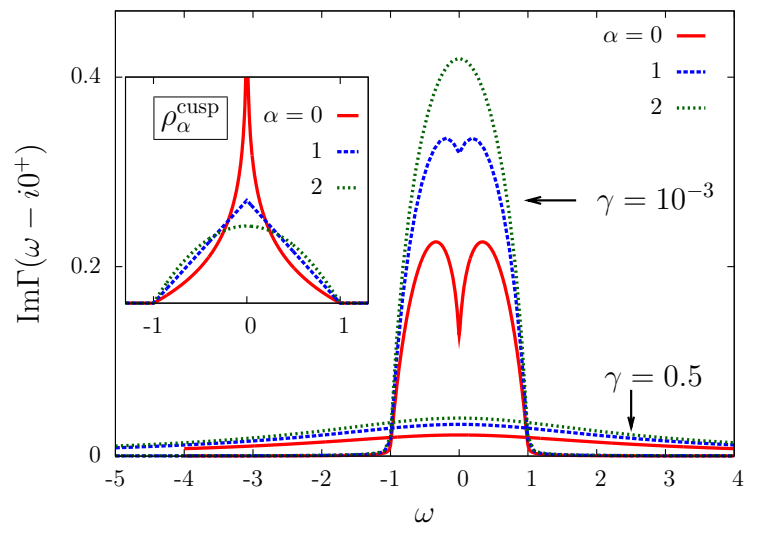

FIG. 1: Imaginary parts of the effective media $\Gamma\left(\omega-i 0^{+}\right)$ [see Eqs. (9), (10) and (11)] as functions of energy $\omega$ for the cases $\alpha=0,1,2$ and two values of the residual interaction $\gamma$. The inset shows the model DOS for the same values of $\alpha$. $W=Z=1$ and $T=\tilde{\epsilon}=0$ was used.

Fermi liquid behavior can be completely destroyed under certain conditions. ${ }^{60-67}$ However, in the present case the effective medium vanishes only logarithmically at the Fermi level, i.e. $\operatorname{Im} \Gamma_{\alpha=0}\left(\omega-i 0^{+}\right) \sim-\frac{1}{\ln |\omega|}$ [see Eq. (A.8)], and therefore the low temperature properties are still expected to be characterized by the strong coupling fixed point as will be shown in Sect. IV D.

The topology of the Fermi surface does not enter the present arguments at any point, only the non-analyticity in the non-interacting DOS does. The latter derives from flat parts in the dispersion relation, which in the case of the $2 d$ square lattice are saddle points at the $X$ points. It is therefore clear, that no nesting property of the Fermi surface, which is present in the square lattice at halffilling, can be responsible for these effects.

\section{SQUARE LATTICE}

\section{A. Half-filling}

In all calculations of this work the hopping matrix element $t$ is used as the energy scale and energies are measured in units $2 t=1$, setting the effective half bandwidth $W=2 d t=2$. In this section the next-nearest neighbor hopping is set to zero, $t^{\prime}=0$. The non-interacting DOS of this $2 d$ square lattice has the same logarithmic singularity at $\omega=0$ as the model DOS of Eq. (8) for $\alpha=0$.

Results of a DMFT(ENCA) calculation for the halffilled $n=1$ Hubbard model on a square lattice are shown in Fig. 2 for various temperatures. In part (a) the noninteracting DOS as well as the fully interacting local spectral function $\rho(\omega)=\frac{1}{\pi} \operatorname{Im} G^{(\text {loc })}\left(\omega-i 0^{+}\right)$are displayed for $U=2 W=4$. The well separated Hubbard bands around the ionic level positions $\omega \approx \pm U / 2= \pm 2$ are clearly visible. Upon lowering the temperature the lattice version of
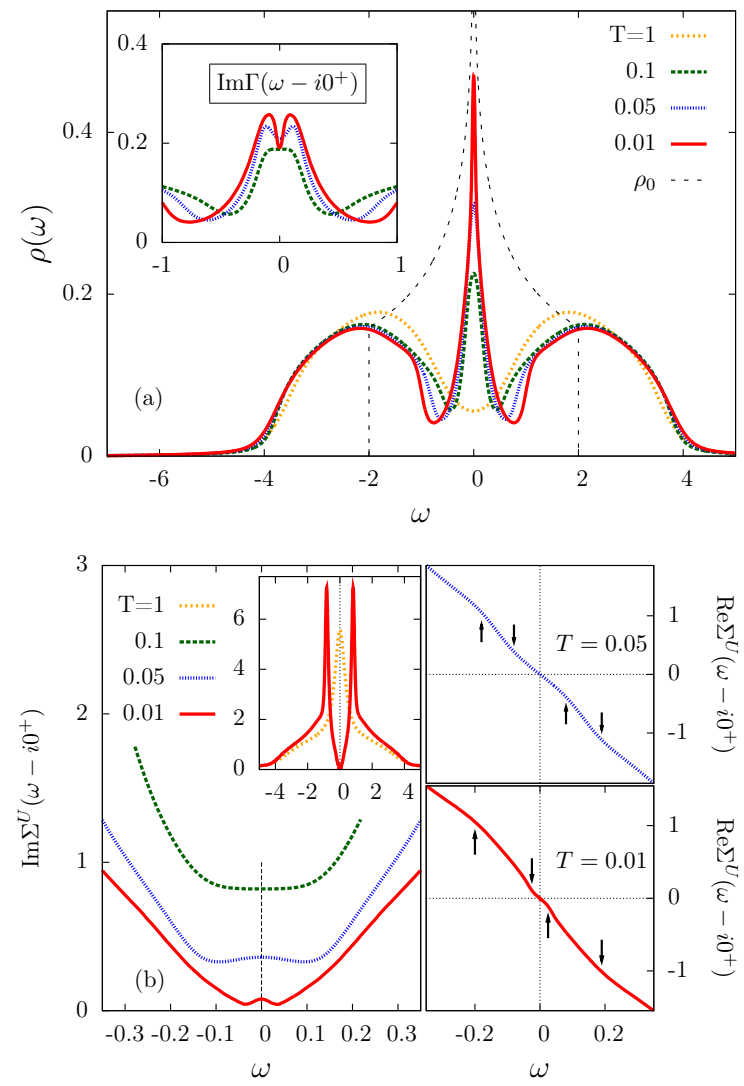

FIG. 2: (a) Local spectral function $\rho(\omega)$ for the symmetric Hubbard model on a $2 d$ square lattice for Coulomb repulsion $U=4(\epsilon=-2)$, half bandwidth $W=2(t=0.5)$ and various temperatures as functions of frequency within DMFT(ENCA). The thin dashed curve represents the noninteracting DOS $\rho_{0}(\omega)$, and the inset shows the imaginary part of the effective medium $\operatorname{Im} \Gamma\left(\omega-i 0^{+}\right)$. (b) Imaginary (left) and real (right) part of the self-energy $\Sigma^{U}\left(\omega-i 0^{+}\right)$for the same parameters as in (a) and $T$ as indicated. The inset of the left panel shows the imaginary part on a wider energy interval. The short arrows in the right graphs point at the approximate positions of the kinks.

the Kondo-effect leads to the build-up of a pronounced many-body resonance at the Fermi level.

The van Hove singularity in $\rho_{0}$ leads to the unusual minimum in the imaginary part of the effective medium $\operatorname{Im} \Gamma\left(\omega-i 0^{+}\right)$[see inset of Fig 2(a)] as it was already found in the previous section. In contrast to Fig. 1 the minimum in $\operatorname{Im} \Gamma\left(\omega-i 0^{+}\right)$is not cusp-like at $\omega=0$ but smeared out due to the imaginary part of the self-energy which is shown in part (b) of the figure. However, the non-analytic structure in the lattice scattering matrix, Eqs. (5) and (9), is sufficient to produce this minimum at low temperatures.

The self-consistency of DMFT leads to an accompanying maximum in $\operatorname{Im} \Sigma^{U}\left(\omega-i 0^{+}\right)$at $\omega=0$, which is in striking contrast to Fermi-liquid behavior $\operatorname{Im} \Sigma^{U}(\omega-$ $\left.i 0^{+}\right) \sim \omega^{2}+\pi^{2} T^{2}$ for small $\omega$ and $T$. The position of 


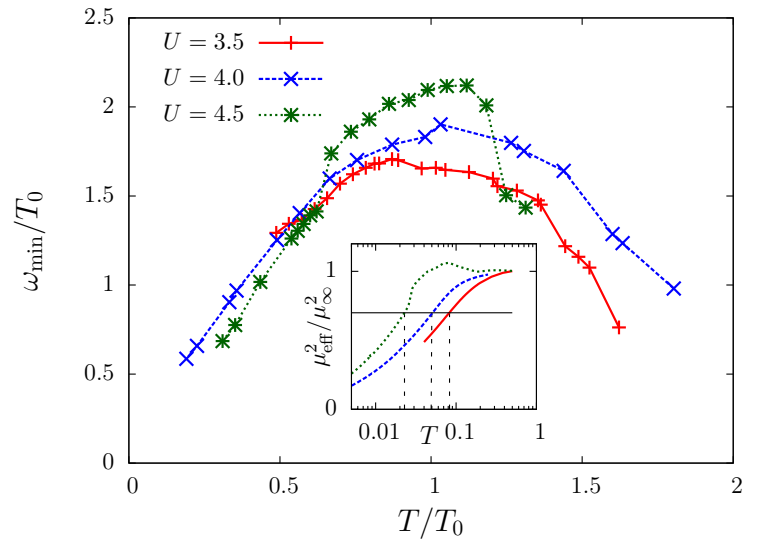

FIG. 3: Position of the minimum $\omega_{\min }$ in $\operatorname{Im} \Sigma^{U}$ in units of the low-energy scale $T_{0}$ as a function of the rescaled temperature $T / T_{0}$ for three different Coulomb repulsions. The inset shows the screened local magnetic moment normalized to its high temperature value $\mu_{\infty}^{2}$ as a function of temperature for the same values of the Coulomb repulsions. The horizontal line indicates the value where $\mu_{\text {eff }}^{2}$ reaches $70 \%$ of $\mu_{\infty}^{2}$ and the vertical dashed lines indicate the low-energy scales: $T_{0}(U=$ $3.5) \approx 0.082, T_{0}(U=4) \approx 0.051$, and $T_{0}(U=4.5) \approx 0.023$.

the two minima emerging at finite frequencies next to the central maximum is moved toward the Fermi level with decreasing temperature so that the anomalous region shrinks.

As a side remark we add here, that we observe additional kinks in the real part of the self-energy as shown in Fig. 2(b), which are associated with this non-Fermi liquid behavior. At energies $\omega \approx \pm 0.2$ the known kinks arising from the coupling of the quasiparticles to local spin fluctuations are observable. ${ }^{68,69}$ The additional kinks are clearly visible at lower energies. In contrast to the kinks at higher energy, their position is temperature dependent and moves toward the Fermi level with decreasing $T$.

Anomalous maxima in $\operatorname{Im} \Sigma^{U}$ are also encountered within the functional renormalization group approach to the Hubbard model at finite temperatures. ${ }^{30,31}$ Crucial for these anomalies to occur is the renormalization of the two-particle interaction vertex. Within DMFT all local $n$-particle vertices and their renormalizations are included.

We extract the low-energy scale $T_{0}$ as the temperature where the effective screened local moment as calculated from the local magnetic susceptibility via ${ }^{50} \mu_{\text {eff }}^{2}=T \chi_{\text {loc }}^{\mathrm{mag}}$ is reduced to $70 \%$ of its high temperature value $\mu_{\infty}^{2}$ (see inset of Fig. 3). This yields the equivalent result to the width of the many-body resonance at the Fermi level in the momentum resolved spectral function $\operatorname{Im} G\left(t_{\underline{k}_{F}}, \omega-\right.$ $\left.i 0^{+}\right) / \pi$ (not shown).

The temperature evolution of the anomalous doublewell structure is governed by the low-energy scale as revealed by Fig. 3. There, the position of the anomalous minimum $\omega_{\min }$ in $\operatorname{Im} \Sigma^{U}$ as a function of temperature for three values of the Coulomb repulsion is shown (the self- energies as well as the minima at $\pm \omega_{\min }$ are symmetric around $\omega=0$ for half-filling). For all $U, \omega_{\min } / T_{0}$ exhibits a dome-shaped curve with both, maximum position and height determined by $T_{0}$. The physical origin can therefore be directly linked to the emergence of the low-energy quasiparticles. At high temperatures the system is dominated by the incoherent charge and spin excitations and the van Hove singularity is concealed. At temperatures of the order of $T_{0}$ the lattice version of the Kondo effect leads to the screening of local magnetic moments. The break-up of screened magnetic moments gives rise to long-lived low-energy quasiparticle excitations which manifest themselves in the many-body resonance at the Fermi level as observed in the spectral function of e.g. Fig. 2(a). But their evolution into the coherent Fermi liquid quasiparticle is disturbed by the enhanced scattering due to the van Hove singularity. This leads to the maximum in $\operatorname{Im} \Sigma^{U}$ at the Fermi level. Further lowering the temperature the phase space volume for quasiparticle scattering shrinks and so does the extension of the maximum.

\section{B. Finite doping}

The van Hove singularity is shifted away from the Fermi energy $\omega=0$ when doping the system, $n=1-\delta$. The corresponding anomalous minimum in the effective medium $\operatorname{Im} \Gamma$ and the maximum in $\operatorname{Im} \Sigma^{U}$ are too moved to finite energies. This can be seen in Fig. 4, where the spectral function, the self-energy, and the effective medium are shown for the Hubbard model on a $2 d$ square lattice $\left(t^{\prime}=0\right)$ with $U=6$ and $\delta=0.02$ (filling $n=0.98$ ).

The quasiparticle peak around $\omega=0$ in the spectral function, as displayed in the inset of Fig. 4(a), acquires a pronounced asymmetry due to the double-well structure in $\operatorname{Im} \Sigma^{U}$. The shoulder in the curve for the lowest temperature might even be interpreted as a precursor of a pseudogap.

The flat parts in the dispersion relation are located energies away from the Fermi level and the dispersion relation can again be approximated linearly in a vary narrow region around the Fermi surface. In this region the usual arguments of microscopic Fermi liquid theory hold and the system forms a regular Fermi liquid at very low temperatures. This is observed in the self-energy shown in Fig. 4(b) where a quadratic minimum forms at $\omega=0$ at $T=0.007$. However, the Fermi liquid parameters will be strongly renormalized as compared to a situation without the van Hove singularity.

\section{Self-consistent treatment with a model DOS}

No true divergence in the non-interacting DOS is necessary for the anomalies described above to occur. A non-analytic cusp as in the model DOS of Eq. (8) for $0<\alpha<2$ is sufficient. This is illustrated in Fig. 5 where 

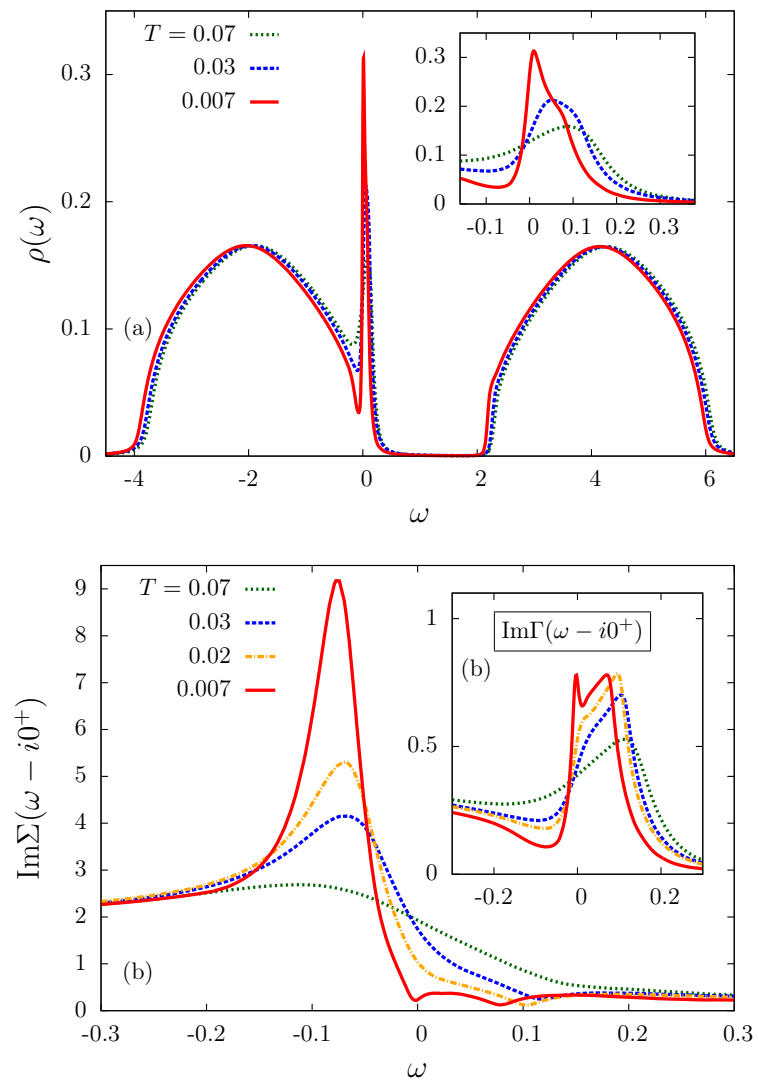

FIG. 4: (a) Local DOS for the asymmetric Hubbard model on a $2 d$ square lattice with $U=6$ and filling $n=0.98$ for various temperatures obtained with the DMFT(ENCA). The inset shows the low-energy region. (b) Imaginary part of the self-energy around the Fermi level for the same parameters as in (a) and temperatures as indicated. The inset shows the imaginary part of the effective medium in the low-energy region.

fully self-consistent DMFT calculations for several model DOS are considered. The first two plots (a) and (b) are for $\rho_{\alpha}^{\text {cusp }}(\omega)$ with $\alpha=1$ at and away from half-filling, respectively. Figures (c) and (d) are for asymmetric DOS which are constant below the Fermi level and have linear (c) and a square-root (d) cusp at $\omega=0$. The imaginary parts of the effective media and self-energies show the characteristic minima and maxima induced by the nonanalyticities in $\rho_{0}$. For the half-filled cases (a), (c) and (d) the anomalies are situated at the Fermi level, while for finite hole doping as in (b) these are moved to positive energies.

\section{Zero Temperature}

Figure 6(a) displays the spectral function for the Hubbard model on a square lattice with $U=4$ at zero temperature and for different doping $\delta$. These spectra were obtained with the $\mathrm{NRG}^{56}$ as impurity solver.
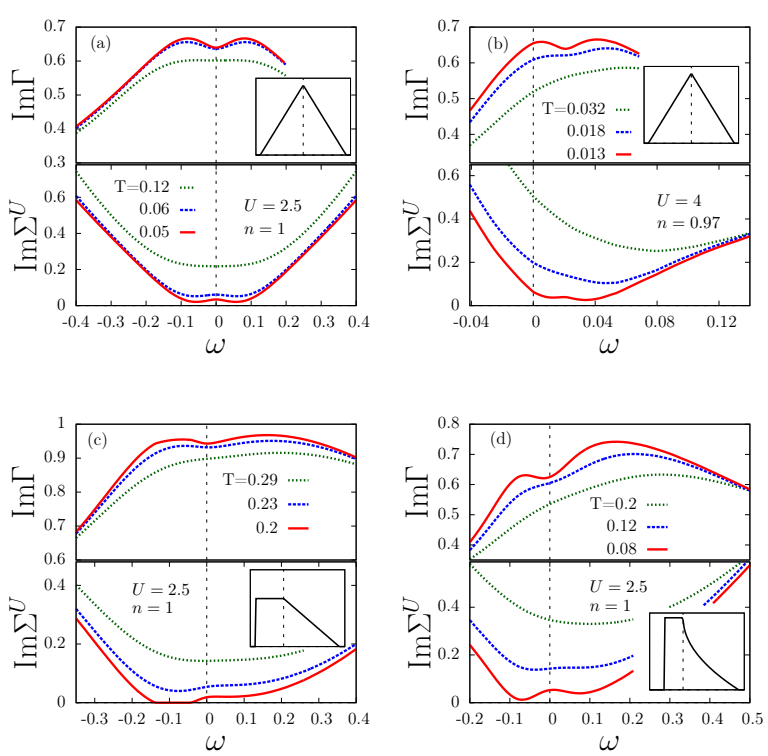

FIG. 5: The imaginary parts of the effective medium (upper graphs) and self-energy (lower graphs) of the Hubbard model within DMFT(ENCA) for different non-interacting DOS, which are shown in the insets. The vertical dashed line indicates the position of the Fermi level. The parameters are indicated in the plots.

As the hole-doping is increased, the lower Hubbard band moves toward the Fermi level and eventually merges with the quasiparticle peak. For very large doping the lower Hubbard band is moved above the Fermi level and $\rho(\omega)$ resembles the non-interacting DOS as the system becomes effectively non-interacting.

The position of the quasiparticle peak is attracted to the Fermi level up to considerable values of doping (roughly $\delta \lesssim 0.2$ ). This pinning becomes more visible in the inset, where the position $\omega_{\max }$ of the maximum in $\rho(\omega)$ is plotted as a function of doping $\delta$ for $U=4$ and $U=0$. In the interacting case $\omega_{\max }$ depends quadratically on the doping, $\omega_{\max } \sim \delta^{2}$, and is considerably reduced compared to $U=0$. The many-body correlations renormalize the van Hove singularity toward the Fermi level especially at small doping $\delta \lesssim 0.2$.

The imaginary part of the self-energy shown in Fig. 6(b) does not display any anomalous double-well structure. The logarithmic van Hove singularity in $\rho_{0}$ is a rather weak divergence and at zero temperature the usual arguments leading to a vanishing self-energy at the Fermi level apply. ${ }^{70}$ However, $\operatorname{Im} \Sigma^{U}$ shows an anomalous frequency dependence at low energies, as it grows like $\operatorname{Im} \Sigma^{U}\left(\omega-i 0^{+}\right) \sim|\omega|^{3 / 2}$ which was also previously found in perturbation theory. ${ }^{20,2190}$ The upper inset reveals this low energy behavior more clearly in a double logarithmic plot.

The quasiparticle peak at the Fermi level for half-filling $\delta=0$ shows a logarithmic divergence as was already observed recently. ${ }^{71}$ But in contrast to what was stated 

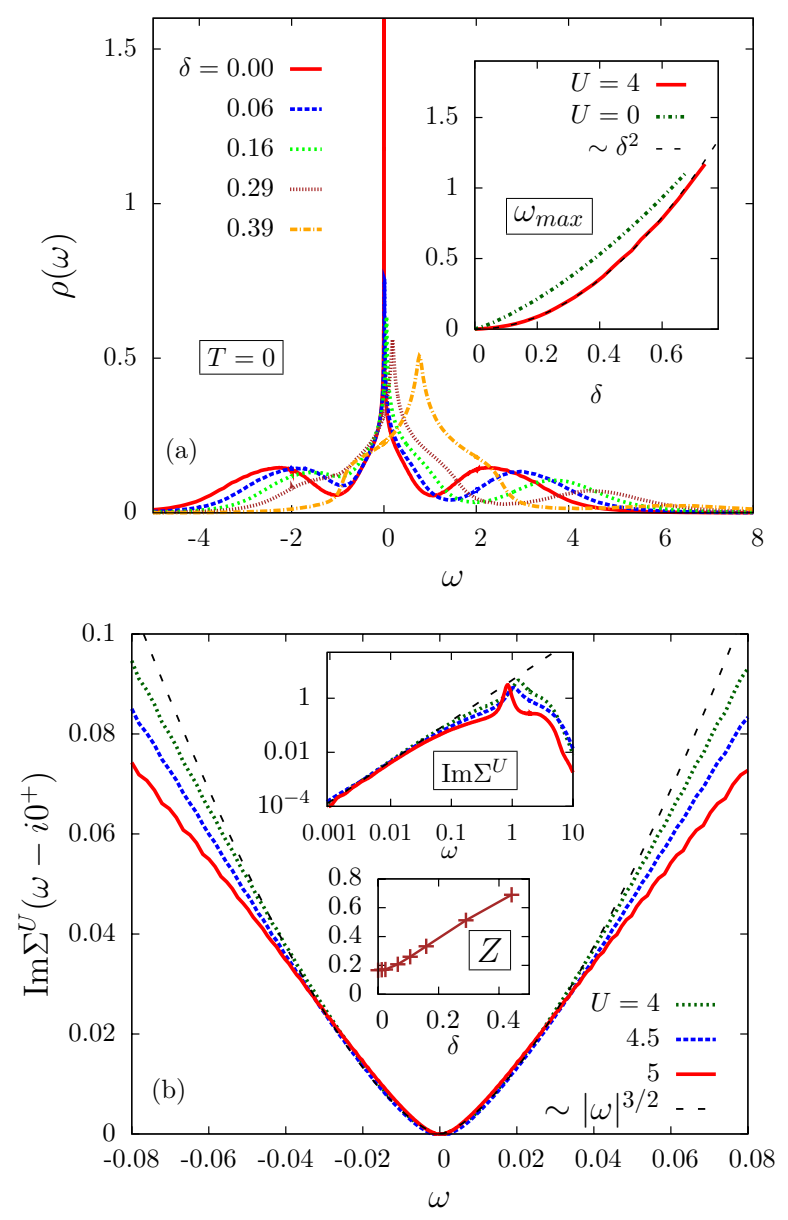

FIG. 6: (a) DMFT(NRG) spectral function at $T=0$ for the Hubbard model on a $2 d$ square lattice for $U=4$ and varying hole doping. The inset shows the position of the maximum as a function of doping for $U=4$ and $U=0$. The dashed line is a fit $\omega_{\max }=2.2 \delta^{2}$. (b) Imaginary part of the self-energy at half-filling $\delta=0$ for three values of the Coulomb repulsion $U$. The curves for $U=4.5$ and $U=5$ were rescaled in order to lie on top of the $U=4$ curves at low $\omega$. The dashed line is a fit with a function $a|\omega|^{3 / 2}$. The upper inset shows the low energy behavior in a double-logarithmic plot. The lower inset shows the quasiparticle weight for $U=4$ as a function of doping.

in Reference 71 , the quasiparticle weight

$$
Z=\frac{1}{1-\left.\frac{\partial \operatorname{Re} \Sigma^{U}}{\partial \omega}\right|_{\omega=0}}
$$

does not vanish at half-filling in our calculation, as can be seen in the inset of Fig. 6(b). (The quasiparticle weight in Fig. 3 of Ref. 71 also seems to extrapolate to a finite value at half-filling but a contradicting statement is made in the text of that reference.) The slope of the real part of the self-energy does consequently not diverge at the Fermi level. This is in accord with the low frequency dependency of the imaginary part $\sim|\omega|^{3 / 2}$ from which the real part is obtained via Kramers-Kronig relation,
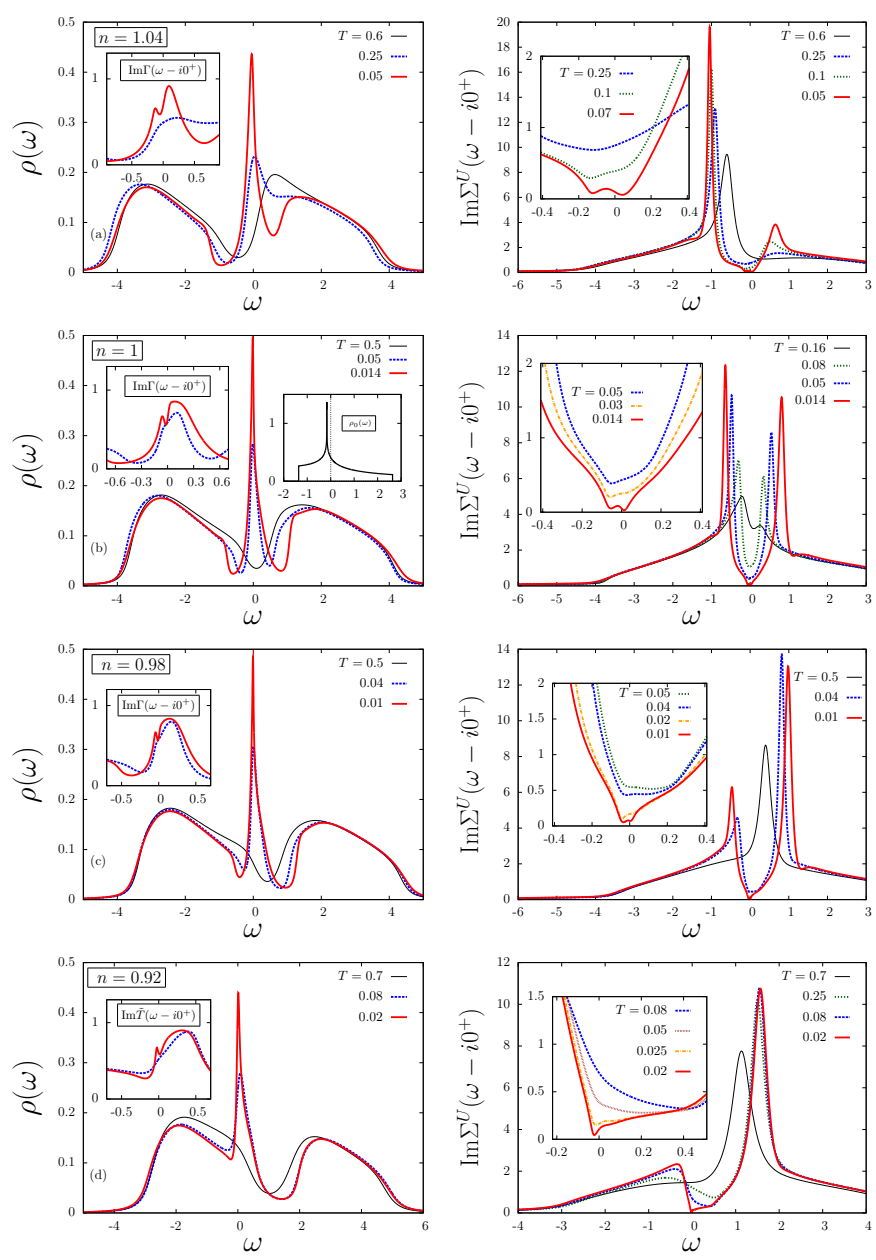

FIG. 7: DMFT(ENCA) spectral functions $\rho(\omega)$ (left panels) and imaginary parts of the self-energy $\Sigma^{U}\left(\omega-i 0^{+}\right)$(right panels) for different filling $n$ for the Hubbard model on the square lattice with additional next-nearest neighbor hopping $t^{\prime}=-0.2 t=-0.1$ and $U=4.25$. The insets in the left panels show the imaginary part of the effective medium while the insets on the right show a close-up of the low-energy region of $\operatorname{Im} \Sigma^{U}$. The non-interacting DOS is shown in the inset for the half-filled case (b).

$\operatorname{Re} \Sigma^{U}\left(\omega-i 0^{+}\right) \sim \omega[\sqrt{\omega}(\operatorname{acoth} \sqrt{\omega}+\operatorname{arccot} \sqrt{\omega})-2]$.

The system is therefore well characterized by a generalized Fermi liquid ${ }^{61-63}$ where $\operatorname{Im} \Sigma^{U}$ shows some anomalous frequency dependence, but still vanishes at the Fermi level and the quasiparticle weight remains finite.

\section{NEXT-NEAREST NEIGHBOR HOPPING}

\section{A. Spectral function and self-energy}

In this section we extend the study to an additional next-nearest neighbor hopping $t^{\prime}=-0.2 t$. The local spectral functions and self-energies displayed in Fig. 7 for $U=4.25$, various fillings and temperatures show pro- 
nounced particle-hole asymmetries.

At low temperatures the anomalous minima in $\operatorname{Im} \Gamma$ and corresponding maxima in the self-energy $\operatorname{Im} \Sigma^{U}$ can be observed slightly below the Fermi level for filling $n=$ $1.04, n=1$, and $n=0.98$. This correlates with the logarithmic van Hove singularity in $\rho_{0}$ shifted below the Fermi level, as displayed in the right inset of Fig. 7(b). For decreasing filling (increasing hole doping) the van Hove singularity is moved toward the Fermi level and the anomalous structures in $\operatorname{Im} \Sigma^{U}$ and $\operatorname{Im} \Gamma$ narrow.

For the largest hole doping $n=0.92$ the imaginary part of the self-energy exhibits only one narrow minimum at very low temperature. Within DMFT the Fermi surface does not change upon increasing the interaction $U,{ }^{9}$ due to the momentum independence of the self-energy. The filling at which the van Hove singularity moves across the Fermi level can be calculated with the non-interacting DOS to be $n \approx 0.82$, which is much less than $n=0.92$ Therefore a double-well structure would be expected but is not observed. The reason is found in the lower Hubbard band reaching the Fermi level. A growing number of empty lattice sites are created and disturb the quasiparticle formation. Additional quasiparticle scattering results and conceals the effect of the van Hove singularity.

Due to the pinning of the singularity to the neighborhood of the Fermi level its influence is strong for all values of filling.

The anomalies correlate with the low-energy scale and occur at temperatures following the trend given by $T_{0}$ as function of filling $n$. $T_{0}$ reaches a minimum for $n \approx 0.98$ to 1 where correlations are strongest and the system is closest to the Mott insulator.

\section{B. Transport}

The anomalous behavior found in the self-energy affects the temperature dependence of transport properties. The static quasiparticle scattering rate at the Fermi level $\operatorname{Im} \Sigma^{U}\left(-i 0^{+}\right)$is shown in Fig. 8(a) as a function of temperature for different filling.

For a $2 d$ Fermi liquid the expected temperature dependence of the scattering rate is given by $\operatorname{Im} \Sigma^{U}\left(-i 0^{+}, T\right) \sim$ $T^{2} \ln (1 / T)$. This behavior is found for low temperatures at half-filling [lowest curve in Fig. 8(a)] and above, i.e. $n \gtrsim 1$ (not shown). Hole-doping induces qualitative changes in the low temperature behavior since the van Hove singularity is moved toward the Fermi level. For fill$\operatorname{ing} n=0.98$ and $n=0.96$ power-law behavior with an exponent close to one is observed, $\operatorname{Im} \Sigma^{U}\left(-i 0^{+}, T\right) \sim T^{1.1}$. For large hole doping, $n=0.92$, the scattering rate is again found to be in accord with usual Fermi liquid variation, $\sim T^{2} \ln (1 / T)$. This is expected to hold for even larger hole-doping $n \lesssim 0.92$, but could not be checked within the present approach due to restriction of the ENCA to relatively high temperatures at large doping.

We want to emphasis that the van Hove singularity induces qualitative changes in the asymptotic low temper-
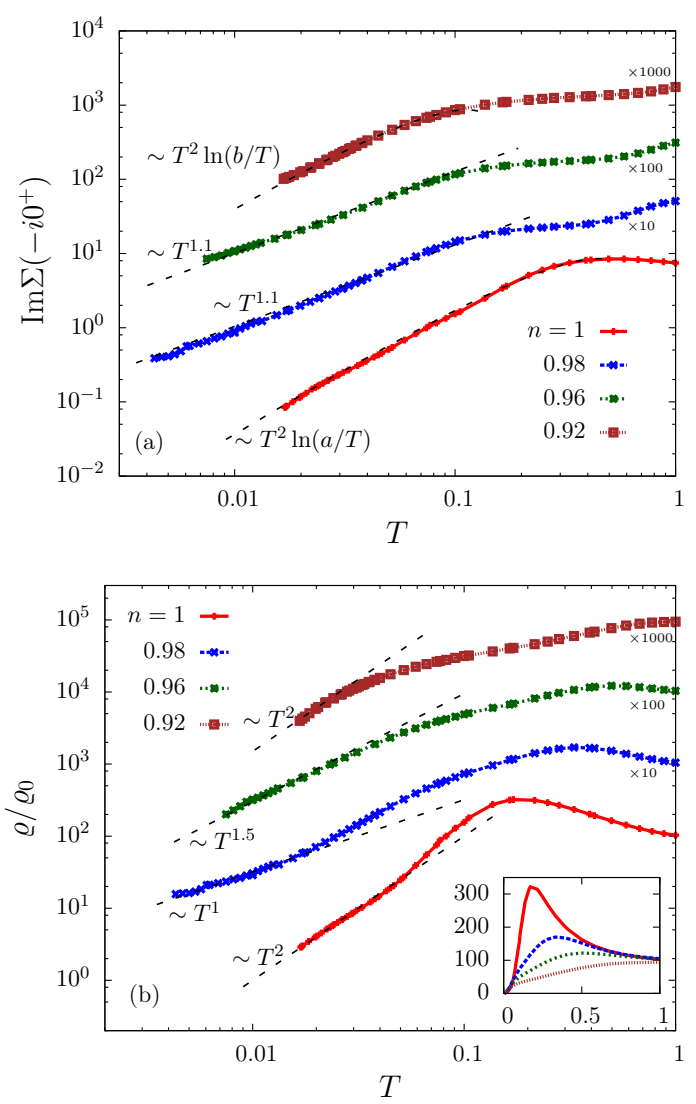

FIG. 8: (a) Static quasiparticle scattering rate $\operatorname{Im} \Sigma^{U}\left(-i 0^{+}\right)$ for the Hubbard with $U=4.25$ on a square lattice with nextnearest neighbor hopping $t^{\prime}=-0.2 t$ as function of temperature in log-log plot. The curves for different filling are multiplied by the factors indicated on the right. (b) Resistivity $\varrho$ (normalized to $\varrho_{0}$ ) for the same parameters as in (a). The inset shows $\varrho$ without the scaling factors and in a nonlogarithmic plot. The dashed lines represent fits with the indicated functions in both plots.

ature behavior of the scattering rate when moved closer to the Fermi surface by doping. $\operatorname{Im} \Sigma^{U}\left(-i 0^{+}, T\right)$ decreases much slower with temperature for fillings around $n \approx 0.96$ when the double-well structure is found in the vicinity of the Fermi level.

With the self-energy at hand one can also calculate transport coefficients. No vertex corrections occur in DMFT due to the momentum independence of the selfenergy and two-particle vertex. ${ }^{72-74}$ The current-current correlation function is completely determined by the particle-hole bubble. Transport quantities can be expressed in terms of a generalized transport lifetime (see, e.g., Ref. 8 or 12 )

$$
L_{\alpha \beta}=\int d \omega\left(-\frac{\partial f(\omega)}{\partial \omega}\right)\left[\tau_{x x}(\omega)\right]^{\alpha} \omega^{\beta-1}
$$

where $f(\omega)=1 /\left(e^{\omega / T}+1\right)$ is the Fermi function. The 
generalized transport lifetime is given by

$$
\begin{aligned}
\tau_{x x}(\omega) & =\frac{1}{N \pi^{2}} \sum_{\underline{k}}\left(\frac{\partial t_{\underline{k}}}{\partial k_{x}}\right)^{2}\left[\operatorname{Im} G\left(t_{\underline{k}}, \omega-i 0^{+}\right)\right]^{2} \\
& =\frac{1}{\pi^{2}} \int d \epsilon \tilde{\rho}_{0}(\epsilon)\left[\operatorname{Im} G\left(\epsilon, \omega-i 0^{+}\right)\right]^{2} .
\end{aligned}
$$

The function

$$
\tilde{\rho}_{0}(\epsilon)=\frac{1}{N} \sum_{\underline{k}}\left(\frac{\partial t_{\underline{k}}}{\partial k_{x}}\right)^{2} \delta\left(\epsilon-t_{\underline{k}}\right)
$$

can be calculated for the cubic lattices and turns out to be a smooth function where the singularities of $\rho_{0}$ are removed due to the derivative.

Only in the Fermi liquid regime is $\tau_{x x}(\omega) \sim$ $1 / \operatorname{Im} \Sigma^{U}\left(\omega-i 0^{+}\right)$and the linearized Boltzmann theory is recovered..$^{75}$

The resistivity

$$
\varrho=\frac{\varrho_{0}}{L_{11}}
$$

with $\varrho_{0}=\frac{2 \hbar a}{\pi e^{2}}$ ( $a$ is the lattice spacing, $e$ the electronic charge) is shown in Fig. 8(b) for different filling. Typical characteristics of strongly correlated systems can be observed for not too large doping. The resistivity $\varrho$ changes from insulating (or semi-conducting) behavior at high temperatures to metallic behavior at low temperatures due to the emergence of (coherent) low-energy quasiparticles (see inset). ${ }^{12}$ The high-temperature resistivity is much larger than the value expected from the Ioffe-Regel condition valid for usual metals, where $\varrho$ is bound by a minimal scattering length of the order of the lattice spacing. ${ }^{76}$ At intermediate temperatures $0.1 \lesssim T \lesssim 0.5$ linear regimes with increasing slope for decreasing doping can be recognized, in accord with earlier studies. ${ }^{8,10}$

The log-log plot (main panel) reveals that the low temperature Fermi liquid behavior $\varrho(T) \sim T^{2}$ is found for $n=1$ and above (not shown). ${ }^{91} \mathrm{~A} T^{2}$-dependency is also observed for $n=0.92$. But for the intermediate values $n=0.98$ and $n=0.96$ a qualitative different behavior is found. $\varrho(T)$ more closely resembles power-laws with exponents 1 and 1.5, respectively. The van Hove singularity is close to the Fermi level and the enhanced scattering increases the resistivity.

In summary, the interplay between the reduction of phase space volume for scattering and the qualitative changes in the frequency dependence of the self-energy within the temperature window causes the qualitative changes in the transport properties.

\section{RELEVANCE FOR THE THEORY OF CUPRATE SUPERCONDUCTORS}

The $t-t^{\prime}$-Hubbard model might be suitable to describe the low-energy physics of cuprate superconductors. Even

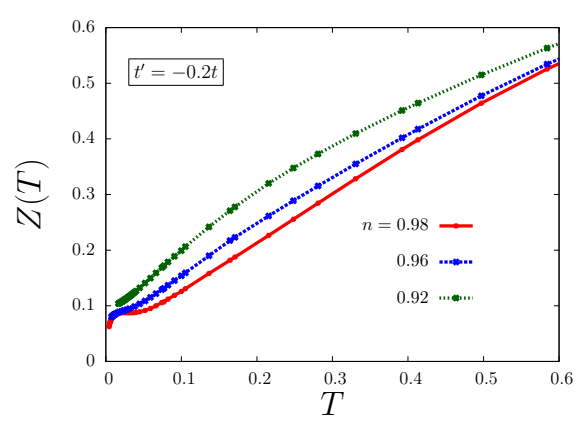

FIG. 9: Generalized quasiparticle weight for the Hubbard model on a square lattice with next-nearest neighbor hopping $t^{\prime}=-0.2 t$ and $U=4.25$ as function of temperature for three different filling obtained from DMFT(ENCA) calculations and Eq. (20).

though DMFT is non-perturbative and includes nontrivial local many-body correlations, it represents a rather poor approximation for the two-dimensional cuprate layers due to the neglect of nonlocal correlations. Therefore, we want to point out that we neither try to model the cuprate superconductors nor can our results be directly transferred to those systems.

However, even in more appropriate theories like cluster-DMFT ${ }^{40}$ the van Hove singularities associated with the lattice structure are retained and therefore the findings of this work have some bearings on the pseudogap and strange metal phase of cuprate superconductors. In the following we will elaborate on some of these aspects.

The van Hove singularity provides an additional mechanism for kinks ${ }^{68,69,77-81}$ in the real part of the selfenergy [see Fig. 2(b)]. The location of the kinks is temperature dependent which should make it possible to discriminate these type of kinks in experiment or calculations.

The non-Fermi liquid self-energy induces an unusual temperature dependence of the generalized quasiparticle weight $^{82}$

$$
\begin{aligned}
Z(T) & =\frac{1}{1-\frac{\operatorname{Im} \Sigma^{U}\left(i \omega_{0}\right)}{\omega_{0}}} \\
& =\frac{1}{1+\int \frac{d \omega}{\pi} \frac{\operatorname{Im} \Sigma^{U}\left(\omega-i 0^{+}\right)}{\omega^{2}+(\pi T)^{2}}},
\end{aligned}
$$

where $\omega_{0}=\pi T$ is the smallest fermionic Matsubara frequency (The form (19) is useful when the self-energy is obtained only at the imaginary Matsubara frequencies like in quantum Monte-Carlo calculations). The generalized quasiparticle weight as calculated with Eq. (20) is shown in Fig. 9 for the Hubbard model on the square lattice with next-nearest neighbor hopping as function of temperature and three different filling. All curves show a change in curvature at the temperature where the anomalous double-well structure emerges. This behavior closely resembles that found in Ref. 83 for a crossover from a 
marginal Fermi liquid at high temperatures to a Fermi liquid at low $T$. Since the self-energies do not show any signs of marginal Fermi liquid behavior (see the previous sections) this reveals a difficulty when interpreting imaginary Matsubara data at finite temperatures. Due to the integral in Eq. (20) the temperature dependence of $Z(T)$ is not determined by the asymptotic low-frequency form of $\operatorname{Im} \Sigma^{U}\left(\omega-i 0^{+}\right)$but by its average spectral weight in the temperature window $|\omega| \lesssim \pi T$. Therefore, the discrimination between different characteristic low-energy forms of $\Sigma^{U}\left(\omega-i 0^{+}\right)$given the self-energy at imaginary Matsubara frequencies seems rather delicate at finite temperature.

The partial destruction of the Fermi surface (Fermi arcs) and the emergence of a pseudogap ${ }^{84-86}$ is of current interest. These phenomena are usually explained to arises from the coupling to strong nonlocal antiferromagnetic correlations. ${ }^{41-44}$ In the present study no nonlocal fluctuations are included but instead the van Hove singularity produces similar features such as a non-Fermi liquid maximum in $\operatorname{Im} \Sigma^{U}\left(\omega-i 0^{+}\right)$and the concomitant reduction of spectral weight [see Fig. 4(a)], However, a true pseudogap is not observed in our approach indicating the importance of non-local fluctuations.

The existence of Fermi arcs and the asymmetry for momentum vectors along the nodal $\underline{k}=\lambda(\pi, \pi)(0 \leq \lambda \leq 1)$ and anti-nodal $\underline{k}=\lambda(0, \pi)$ direction is natural in the present scenario. The flat parts in the dispersion relation occur at the $X$ point in the Brillouin zone where the lowtemperature quasiparticles are most strongly affected and consequently the Fermi liquid description breaks down there first. ${ }^{30,31}$ Also the stability of the induced nonFermi liquid behavior up to a doping of the order of $20 \%$ which was encountered in other studies ${ }^{41}$ can be explained by the pinning of the van Hove singularity to the Fermi level.

Unarguably, nonlocal correlations are vital for the understanding of cuprate superconductors. But as the above aspects suggest, some of the non-Fermi liquid signatures can be produced - or at least aided - by the presence of a van Hove singularity in the vicinity of the Fermi surface. This is especially important for lattices without nesting or with frustration, where nonlocal antiferromagnetic spin-fluctuations are usually suppressed and thus might be too weak to produce non-Fermi liquid signatures. Therefore, we argue that the interpretation solely in terms of collective modes which couple to the electronic degrees of freedom is too simplified and the influence of the saddle points in the Brillouin zone has to be reconsidered.

\section{CONCLUSIONS}

In the present work we have focused on the influence of a van Hove singularity in the non-interaction density of states on the low temperature properties of the Hubbard model. In order to include non-perturbative correlation effects we used the DMFT to calculate the spectral function and self-energy. As impurity solvers we employed the enhanced non-crossing approximation for finite temperatures $T>0$ and the numerical renormalization group for $T=0$. Both methods yield dynamic quantities directly on the real frequency axis, which avoids the inaccuracies connected with a numerical analytic continuation of imaginary time data.

The van Hove singularity causes profound changes of the low-energy Fermi liquid properties usually encountered within DMFT for the Hubbard model. For the strongly correlated metal close to the Mott Hubbard metal-insulator transition the imaginary part of the selfenergy develops an unusual double-well structure at finite temperatures. This anomalous structure appears at temperatures on the order of the many-body scale $T_{0}$ which also determines its energy spread. It originates from an enhanced scattering of the low-energy quasiparticles at the saddle points in the dispersion relation associated with the van Hove singularity.

Using a model DOS, we have shown analytically that the non-Fermi liquid signature in the quasiparticle selfenergy is directly linked to non-analytic logarithmic contributions to the lattice scattering matrix. As a consequence the medium for the effective impurity develops a dip at the van Hove singularity. In case of a logarithmically diverging non-interacting DOS this dip produces a soft-gap in the effective medium at $T=0$.

At zero temperature the interacting spectral function for a square lattice exhibits a logarithmic divergence for half-filling. The system is well characterized by a generalized Fermi liquid where the quasiparticle weight remains finite and the imaginary part of the self-energy vanishes at the Fermi level. But the frequency dependent scattering rate increases with a non-Fermi liquid exponent $\approx 1.5 \neq 2$. Upon doping a pinning of the van Hove singularity to the Fermi level is observed. Its signature in the spectral function is always renormalized toward the Fermi level and stays close to it up to rather large doping $\delta \lesssim 0.2$.

For the square lattice with a finite next-nearest neighbor hopping $t^{\prime}=-0.2 t$ the van Hove singularity in the non-interacting DOS is located below the Fermi level and moved toward it upon increasing hole-doping. This trend is also observed in the anomalous maximum in the selfenergy.

In case of half-filling and electron doping, as well as for large hole doping, the zero-frequency quasiparticle scattering rate exhibits a low temperature dependence which is consistent with that of a two-dimensional Fermi liquid. In between these fillings the decrease is much slower and follows a power law with exponent close to one.

A similar signature of the van Hove singularity was found in the resistivity. A Fermi liquid $T^{2}$ dependence emerged for $n \gtrsim 1$ and $n \lesssim 0.92$, while for $0.92 \lesssim n \lesssim 1$ the resistivity was enhanced and a decrease with exponents less than two was observed.

The findings of this work bear some implications on 
theories for cuprate superconductors. Even thought no nonlocal fluctuations were included, we still produced qualitative features usually only obtained within more advanced theories where those correlations are incorporated. This raises the questions concerning the origin and physical mechanism behind such features and we think the role of the van Hove singularity in connection with strong correlations should be further explored in the future.

\section{Acknowledgments}

The author acknowledges very fruitful discussions with N. Grewe, F. B. Anders, T. Jabben, E. Jakobi and F. Güttge. The author especially thanks F. B. Anders for providing him with his NRG code with which the zero temperature calculations of this work were done. This work was supported by the Deutsche Forschungsgemeinschaft under Grant No. AN 275/6-2.

\section{Appendix: Details of the model DOS calculation}

The scattering matrix can be calculated analytically via Eq. (5) for any $\tilde{G}$ (omitting the arguments),

$$
\begin{aligned}
T & =\int_{-W}^{W} d x \frac{x^{2} \rho_{\alpha}^{\text {cusp }}(x)}{\tilde{G}^{-1}-x} \\
& \stackrel{\alpha \geq 0}{=} \frac{1+\alpha}{\alpha} \frac{1}{\tilde{G}}\left[-1+\frac{1}{\tilde{G} W} \operatorname{atanh}(\tilde{G} W)\right. \\
& \left.-\frac{(\tilde{G} W)^{2}}{3+\alpha}{ }_{2} \mathrm{~F}_{1}\left(1, \frac{3+\alpha}{2} ; \frac{5+\alpha}{2} ;(\tilde{G} W)^{2}\right)\right] \\
& \stackrel{\alpha=0}{=} \frac{1}{\tilde{G}}\left[-1+\frac{\operatorname{Li}_{2}(\tilde{G} W)-\operatorname{Li}_{2}(-\tilde{G} W)}{2 \tilde{G} W}\right],
\end{aligned}
$$

where ${ }_{2} \mathrm{~F}_{1}$ is the Gauss's hypergeometric function and $\mathrm{Li}_{2}$ the Dilogarithm. Equation (4) then yields the effective medium

$$
\begin{aligned}
\Gamma \stackrel{\alpha \geq 0}{=} \frac{1}{\tilde{G}}-\frac{W \alpha}{(1+\alpha) \operatorname{atanh}(\tilde{G} W)-\tilde{G} W-\frac{1+\alpha}{3+\alpha}(\tilde{G} W)^{3}{ }_{2} \mathrm{~F}_{1}\left(1, \frac{3+\alpha}{2} ; \frac{5+\alpha}{2} ;(\tilde{G} W)^{2}\right)} \\
\stackrel{\alpha=0}{=} \frac{1}{\tilde{G}}+\frac{2 W}{\operatorname{Li}_{2}(-\tilde{G} W)-\operatorname{Li}_{2}(\tilde{G} W)} .
\end{aligned}
$$

For $\alpha=0$, Eq. (9) already constitutes the final expression, while for the $\alpha=1,2$ equation (A.4) can be further simplified.

For $\alpha=1$ the hypergeometric function reduces to

$$
{ }_{2} \mathrm{~F}_{1}\left(1,2 ; 3 ; z^{2}\right)=-\frac{2}{z^{4}}\left[z^{2}+\ln \left(1-z^{2}\right)\right],
$$

leading to the effective medium displayed in Eq. (10). In case of $\alpha=2$

$$
{ }_{2} \mathrm{~F}_{1}\left(1, \frac{5}{2} ; \frac{7}{2} ; z^{2}\right)=-\frac{5}{3} \frac{1}{z^{5}}\left[3 z+z^{3}-3 \operatorname{atanh}(z)\right],
$$

yields the effective medium of Eq. (11).

Assuming a Fermi liquid as in Eq. (12) and taking the limit $\gamma \rightarrow 0$ the effective media take the approximate low-energy forms, $\omega \ll W=1(Z=1, T=0, \tilde{\epsilon}=0)$

$$
\begin{aligned}
& \operatorname{Im} \Gamma_{\alpha=0}\left(\omega-i 0^{+}\right) \approx-\frac{2}{\pi} \frac{\ln |\omega|}{\frac{\pi^{2}}{4}+(\ln |\omega|)^{2}} \\
& \operatorname{Im} \Gamma_{\alpha=1}\left(\omega-i 0^{+}\right) \approx \frac{\pi(1-|\omega|)}{\pi^{2}(1-|\omega|)^{2}+4 \omega^{2}(1-\ln |\omega|)^{2}} \\
& \operatorname{Im} \Gamma_{\alpha=2}\left(\omega-i 0^{+}\right) \approx \frac{\pi}{3} \frac{1-\omega^{2}}{\frac{\pi^{2}}{4}+\left(4-\frac{\pi^{2}}{2}\right) \omega^{2}}
\end{aligned}
$$

For $\alpha=0$ the effective medium reaches zero at zero energy. For $\alpha=1$ it saturates at a value $1 / \pi$ but the non-analytic cusp still remains, while for $\alpha=2$ the cusp is removed and $\operatorname{Im} \Gamma_{\alpha=2}\left(-i 0^{+}\right)=4 /(3 \pi)$.
* Electronic address: sebastian.schmitt@tu-dortmund.de 1 R. Shankar, Rev. Mod. Phys. 66, 129 (1994).

2 A. A. Abrikosov, L. P. Gorkov, and I. E. Dzyaloshinski, Methods of Quantum Field Theory in Statistical Physics (Prentice-Hall, Englewood Cliffs, USA, 1963).
3 A. Virosztek and J. Ruvalds, Phys. Rev. B 42, 4064 (1990).

4 J. Voit, Rep. Prog. Phys 58, 977 (1995).

${ }^{5}$ C. Varma, Z. Nussinov, and W. van Saarloos, Phys. Rep. 361, 267 (2002).

6 M. Vojta, Rep. Prog. Phys. 66, 2069 (2003). 
7 A. Georges, G. Kotliar, W. Krauth, and M. J. Rozenberg, Rev. Mod. Phys. 68, 13 (1996).

8 T. Pruschke, M. Jarrell, and J. Freericks, Adv. Phys. 44, 187 (1995).

${ }^{9}$ E. Müller-Hartmann, Z. Phys. B 76, 211 (1989).

10 M. Jarrell and T. Pruschke, Phys. Rev. B 49, 1458 (1994).

11 T. Pruschke, D. L. Cox, and M. Jarrell, Phys. Rev. B 47, 3553 (1993).

12 J. Merino and R. H. McKenzie, Phys. Rev. B 61, 7996 (2000).

13 R. Bulla, Phys. Rev. Lett. 83, 136 (1999).

14 A. C. Hewson, The Kondo Problem to Heavy Fermions (Cambridge University Press, Cambridge, 1993).

15 N. Grewe, Ann. Phys. (Leipzig) 14, 611 (2005).

16 N. Grewe, S. Schmitt, T. Jabben, and F. B. Anders, J. Phys.: Condens. Matter 20, 365217 (2008).

17 C. M. Varma, P. B. Littlewood, S. Schmitt-Rink, E. Abrahams, and A. E. Ruckenstein, Phys. Rev. Lett. 63, 1996 (1989).

18 D. M. Newns, P. C. Pattnaik, and C. C. Tsuei, Phys. Rev. B 43, 3075 (1991).

19 D. M. Newns, C. C. Tsuei, P. C. Pattnaik, and C. L. Kane, Comments Condens. Matter Phys. 15, 273 (1992).

20 S. Gopalan, O. Gunnarsson, and O. K. Andersen, Phys. Rev. B 46, 11798 (1992).

21 R. Hlubina and T. M. Rice, Phys. Rev. B 51, 9253 (1995).

22 I. Dzyaloshinskii, J. Phys. I France 6, 119 (1996).

23 R. S. Markiewicz, J. Phys. Chem. Solids 58, 1179 (1997).

24 D. Menashe and B. Laikhtman, Phys. Rev. B 59, 13592 (1999).

25 F. Onufrieva and P. Pfeuty, Phys. Rev. Lett. 82, 3136 (1999).

${ }^{26}$ G. Kastrinakis, Physica C 340, 119 (2000).

27 V. Y. Irkhin and A. A. Katanin, Phys. Rev. B 64, 205105 (2001).

28 A. A. Katanin and A. P. Kampf, Phys. Rev. B 68, 195101 (2003).

29 R. Roldán, M. P. López-Sancho, F. Guinea, and S.-W. Tsai, Phys. Rev. B 74, 235109 (2006).

30 A. A. Katanin and A. P. Kampf, Phys. Rev. Lett. 93, 106406 (2004).

31 D. Rohe and W. Metzner, Phys. Rev. B 71, 115116 (2005).

32 P. Schlottmann, Phys. Rev. B 68, 125105 (2003).

33 W. Jones and N. H. March, Theoretical Solid State Physics (Wiley, London, 1973).

34 V. Y. Irkhin, A. A. Katanin, and M. I. Katsnelson, Phys. Rev. Lett. 89, 076401 (2002).

35 Q. Si and K. Levin, Phys. Rev. B 44, 4727 (1991).

${ }^{36} \mathrm{P}$. Majumdar and H. R. Krishnamurthy, arXiv:condmat/9604057v1 (1996).

37 J. Gonzlez, F. Guinea, and M. A. H. Vozmediano, Nucl. Phys. B 485, 694 (1997).

38 S. Odashima, A. Avella, and F. Mancini, Phys. Rev. B 72, 205121 (2005).

39 A. Macridin, M. Jarrell, T. Maier, and G. A. Sawatzky, Phys. Rev. B 71, 134527 (2005).

${ }^{40}$ T. Maier, M. Jarrell, T. Pruschke, and M. H. Hettler, Rev. Mod. Phys. 77, 1027 (2005).

41 A. Liebsch and N.-H. Tong, Phys. Rev. B 80, 165126 (2009).

42 B. Kyung, S. S. Kancharla, D. Sénéchal, A.-M. S. Tremblay, M. Civelli, and G. Kotliar, Phys. Rev. B 73, 165114 (2006).

43 A. Macridin, M. Jarrell, T. Maier, P. R. C. Kent, and
E. D'Azevedo, Phys. Rev. Lett. 97, 036401 (2006).

44 A. A. Katanin, A. Toschi, and K. Held, Phys. Rev. B 80, 075104 (2009).

45 A.-M. S. Tremblay, B. Kyung, and D. Snchal, Low Temp. Phys. 32, 424 (2006).

46 T. Pruschke and N. Grewe, Z. Phys. B 74, 439 (1989).

47 J. Holm and K. Schönhammer, Solid State Commun. 69, 969 (1989).

48 H. Keiter and Q. Qin, Physica B 163, 594 (1990).

49 N. E. Bickers, Rev. Mod. Phys. 59, 845 (1987).

50 S. Schmitt, T. Jabben, and N. Grewe, Phys. Rev. B 80, 235130 (2009).

51 H. R. Krishna-murthy, J. W. Wilkins, and K. G. Wilson, Phys. Rev. B 21, 1003 (1980).

${ }^{52}$ H. R. Krishna-murthy, J. W. Wilkins, and K. G. Wilson, Phys. Rev. B 21, 1044 (1980).

53 R. Bulla, T. Costi, and T. Pruschke, Rev. Mod. Phys. 80, 395 (2008).

${ }^{54}$ F. B. Anders and A. Schiller, Phys. Rev. B 74, 245113 (2006).

55 F. B. Anders and A. Schiller, Phys. Rev. Lett. 95, 196801 (2005).

56 R. Peters, T. Pruschke, and F. B. Anders, Phys. Rev. B 74, 245114 (2006).

57 V. L. Campo and L. N. Oliveira, Phys. Rev. B 72, 104432 (2005).

58 R. Bulla, T. A. Costi, and D. Vollhardt, Phys. Rev. B 64, 045103 (2001).

59 S. Schmitt, Ph.D. thesis, TU Darmstadt (2008), available at http://tuprints.ulb.tu-darmstadt.de/1264/.

60 R. Bulla, T. Pruschke, and A. Hewson, J. Phys.: Condens. Matter 9, 10463 (1997).

61 C. Gonzalez-Buxton and K. Ingersent, Phys. Rev. B 57, 14254 (1998).

62 D. E. Logan and M. T. Glossop, J. Phys.: Condens. Matter 12, 985 (2000).

63 M. Glossop and D. Logan, Eur. Phys. J. B 13, 513 (2000).

64 K. Ingersent and Q. Si, Phys. Rev. Lett. 89, 076403 (2002).

65 M. T. Glossop and D. E. Logan, J. Phys.: Condens. Matter 15, 7519 (2003).

${ }^{66}$ L. Fritz and M. Vojta, Phys. Rev. B 70, 214427 (2004).

67 L. Fritz, S. Florens, and M. Vojta, Phys. Rev. B 74, 144410 (2006).

68 K. Byczuk, M. Kollar, K. Held, Y.-F. Yang, I. A. Nekrasov, T. Pruschke, , and D. Vollhardt, Nature Physics 3, 168 (2007).

69 C. Raas, P. Grete, and G. S. Uhrig, Phys. Rev. Lett. 102, 076406 (2009).

70 J. M. Luttinger, Phys. Rev. 121, 942 (1961).

71 R. Žitko, J. Bonča, and T. Pruschke, Phys. Rev. B 80, 245112 (2009).

72 A. Khurana, Phys. Rev. Lett. 64, 1990 (1990).

73 H. Schweitzer and G. Czycholl, Phys. Rev. Lett. 67, 3724 (1991).

74 E. Müller-Hartmann, Z. Phys. B 74, 507 (1989).

75 N. W. Ashcroft and N. D. Mermin, Solid State Physics (Saunders College, Philadelphia, 1976).

76 O. Gunnarsson, M. Calandra, and J. E. Han, Rev. Mod. Phys. 75, 1085 (2003).

77 A. Lanzara, P. V. Bogdanov, X. J. Zhou, S. A. Kellar, D. L. Feng, E. D. Lu, T. Yoshida, H. Eisaki, A. Fujimori, K. Kishio, et al., Nature 412, 510 (2001).

78 Z. X. Shen, A. Lanzara, S. Ishihara, and N. Nagaosa, Phil. Mag. B 82, 1349 (2002). 
79 A. Macridin, M. Jarrell, T. Maier, and D. J. Scalapino, Phys. Rev. Lett. 99, 237001 (2007).

80 T. Valla, T. E. Kidd, W.-G. Yin, G. D. Gu, P. D. Johnson, Z.-H. Pan, and A. V. Fedorov, Phys. Rev. Lett. 98, 167003 (2007).

81 J. Graf, G.-H. Gweon, K. McElroy, S. Y. Zhou, C. Jozwiak, E. Rotenberg, A. Bill, T. Sasagawa, H. Eisaki, S. Uchida, et al., Phys. Rev. Lett. 98, 067004 (2007).

82 J. W. Serene and D. W. Hess, Phys. Rev. B 44, 3391 (1991).

83 N. S. Vidhyadhiraja, A. Macridin, C. Sen, M. Jarrell, and M. Ma, Phys. Rev. Lett. 102, 206407 (2009).

${ }^{84}$ M. R. Norman, H. Ding, M. Randeria, J. C. Campuzano, T. Yokoya, T. Takeuchi, T. Takahashi, T. Mochiku, K. Kadowaki, P. Guptasarma, et al., Nature 392, 157 (1998).

85 A. Kanigel, M. R. Norman, M. Randeria, U. Chatterjee, S. Souma, A. Kaminski, H. M. Fretwell, S. Rosenkranz, M. Shi, T. Sato, et al., Nat Phys 2, 447 (2006).

${ }^{86}$ H.-B. Yang, J. D. Rameau, P. D. Johnson, T. Valla, A. Tsvelik, and G. D. Gu, Nature 456, 77 (2008).

87 M. Jarrell, T. Maier, C. Huscroft, and S. Moukouri, Phys. Rev. B 64, 195130 (2001).

88 M. Jarrell, Phys. Rev. B 51, 7429 (1995).

89 S. Schmitt and N. Grewe, Physica B 359-361, 777 (2005).

90 The exponent $3 / 2$ is found in perturbation theory for quasiparticles at the Fermi level, but not at the van Hove singularity, which scatter from quasiparticles at the van Hove singularities. For quasiparticles at the van Hove singularity $\operatorname{Im} \Sigma^{U}\left(\omega-i 0^{+}\right) \sim|\omega|$ and scattering processes not involving the saddle points produce the usual $2 d$ Fermi liquid result $\operatorname{Im} \Sigma^{U}\left(\omega-i 0^{+}\right) \sim \omega^{2} \ln 1 /|\omega|$. DMFT does not resolve the different momentum dependencies in the interaction channels and averages over the whole Brillouin zone. Due to its large phase space, the contribution $\sim|\omega|^{3 / 2}$ dominates this average and is therefore observed in the DMFT. This is confirmed by fitting the imaginary part with a function $A|\omega|^{3 / 2}+B|\omega|$ which yields a very small linear contribution $B / A \approx 1-2 \%$ without changing the range of validity of the fit. The usual $2 d$ Fermi contribution $\sim \omega^{2} \ln 1 /|\omega|$ vanishes too fast for $\omega \rightarrow 0$ to make a significant contribution at low energies.

91 The reason, why we observe a quadratic temperature dependence, which is characteristic for Fermi liquids in dimensions $d>2$ and not the two-dimensional form $\varrho \sim$ $T^{2} \ln (1 / T)^{2}$ is not completely clear to us at present. It is probably related to the neglect of vertex correction within DMFT. Ignoring the momentum dependence in the twoparticle vertex leads to a violation of momentum conservation at internal vertices. ${ }^{87}$ The resultant mean-field decoupling in the Bethe-Salpeter equations ${ }^{59,88,89}$ for the twoparticle current-current correlation function introduces additional averages over the Brillouin zone and mimics an effectively higher dimensionality for transport quantities. 